Relations industrielles

Industrial Relations

\title{
Le Québec, au point tournant de son histoire
}

\author{
Eric W. Kierans
}

Volume 19, numéro 3, juillet 1964

URI : https://id.erudit.org/iderudit/1021280ar

DOI : https://doi.org/10.7202/1021280ar

Aller au sommaire du numéro

\section{Éditeur(s)}

Département des relations industrielles de l’Université Laval

ISSN

0034-379X (imprimé)

1703-8138 (numérique)

Découvrir la revue

Citer ce document

Kierans, E. W. (1964). Le Québec, au point tournant de son histoire. Relations industrielles / Industrial Relations, 19(3), 395-404.

https://doi.org/10.7202/1021280ar
Résumé de l'article

L'auteur, en se plaçant dans l'optique très particulière de l'économiste, décrit d'abord les trois changements qui, selon lui, se produisent dans le Québec. Puis, il les examine de deux points de vue différents : d'une part, du point de vue de l'homme d'affaires d'expression française et, d'autre part, du point de vue des milieux d'affaires d'expression anglaise.
Tous droits réservés (C Département des relations industrielles de l’Université Laval, 1964
Ce document est protégé par la loi sur le droit d'auteur. L’utilisation des services d'Érudit (y compris la reproduction) est assujettie à sa politique d'utilisation que vous pouvez consulter en ligne.

https://apropos.erudit.org/fr/usagers/politique-dutilisation/ 


\title{
INFORMATIONS
}

\section{LE QUEBEC, AU POINT TOURNANT DE SON HISTOIRE *}

\section{Eric W. Kierons}

\begin{abstract}
L'auteur, en se plaçant dans l'optique très particulière de l'économiste, décrit d'abord les trois changements qui, selon lui, se produisent dans le Québec. Puis, il les examine de deux points de vue différents: d'une part, du point de vue de l'homme d'affaires d'expression française et, d'autre part, du point de vue des milieux d'affaires d'expression onglaise.
\end{abstract}

Chocun d'entre nous doit ovoir le souci de déterminer le sens et la signification des changements qui se produisent en ce moment dans la province de Québec. Les modifications que subit notre milieu sont faciles à constater cor elles sont d'ordre physique et matériel: nous pouvons les mesurer en fonction du nombre et de l'importance toujours croissants de nos écoles, de nos collèges, de nos hópitoux, de nos usines, de nos mines et de nos routes. La métomorphose survenant dans notre mentalité est encore plus marquée, quoique mains opparente, à mesure que nos connaissances, nos qualités et notre compétence technique deviennent plus vostes grâce ò l'amélioration du niveau général de l'instruction et ou fait que nous comprenons toujours mieux ce monde dans lequel nous vivons, grâce à ces moyens d'information toujours plus rapides que sont la presse, la radio et la télévision.

Ces chongements sont profonds. A vrai dire, il s'exercent de deux manières: objectivement, sous l'aspect d'une transformation rapide du monde qui nous entoure, et subjectivement, dans le sens que nous summes copobles de percevoir plus intimement et d'appréhender d'une monière plus complète lo nature même de notre conscience.

Si des changements se produisent ou fin fond de nous-mêmes tout autant que dans notre milieu ambiant, il doit $y$ ovoir alors, manifestement, une troisième modification, $c$ savoir celle qui s'exerce dons les relations entre ces deux pôles que sont notre conscience et le mande extérieur. Une telle transformation signifie que les institutions sociales, politiques et économiques, dont les réseoux intimement reliés constituent les structures d'une société, ne sont pas d'une noture immuoble.

C'est ainsi que l'Histoire est en troin de s'ćcrire, en ce moment, dans le Québec, alors que nous cherchons ò déterminer d'une port les nouvelles structures politiques qui sauront libérer l'énergie créatrice de notre jeunesse, et, d'autre port, les nouvelles institutions économiques qui nous permettront de faire face efficacement dons un monde de plus en plus portagé entre un certain nombre de sociétés internationales géontes. Cette recherche, qui se matérialise por des expressions comme "l'Etat du Québec 》, ne représente simplement que le désir naturel et puissant d'un peuple décidé à exprimer la signification nouvelle de so propre existence, d'un peuple qui a pris conscience de l'ampleur toujours pius vaste de sa dimension.

* Causerie prononcée por I'Honorable Eric W. Kierans, Ministre du Revenu pour lo Province de Québec, devant la Chambre de Commerce du District de Montréal, à I'Hôtel Mont-Royal, mardi, le 25 février 1964. 
Se veux tout d'abord souligner le fait que j'oi l'intention de me placer dans l'optique très particulière de l'économiste et que je comprends fort bien que l'historien, le juriste ou le spécialiste en sciences politiques puissent considérer, comme étant beaucoup plus importantes, d'outres caractéristiques de ce nouveau milieu ambiant. Cependant, si chacun d'entre nous cherche dons sa spécialité à procéder à une analyse toujours plus raffinée, du dialogue qui s'ensuivra ne pourro joïlir qu'une compréhension plus complète. Car c'est cette compréhension qui est à la bose même d'une direction efficoce, d'une persuasion productive et, éventuellement, d'une entente valable.

Deuxièmement, si d'une part je vois tout particulièrement insister, dans cette causerie, sur les chongements en cours, celo ne veut pas dire, por contre, que je ne nourris pas un certain respect pour les coutumes, les traditions et les méthodes de nos ancêtres. Nuus devons vénérer notre possé, certes, mois nous ne pouvons pos nous permettre d'être déterminés seulement par notre histoire au point de l'accepter comme la norme de notre avenir, car, alors, nous n'ourions plus d'existence, plus de possibilités, plus de défis qui soient vroiment les nôtres.

Alfred North Whiteheod o écrit dons son oeuvre intitulée «Symbolisme »: *Ces sociétés qui ne sont pas en mesure de combiner le respect dû à leurs symboles ovec la liberté de les réviser doivent en fin de compte tomber en décadence soit sous l'effet de l'anarchie, soit por une lente otrophie qui étouffe leur existence sous le poids d'ombres inutiles. 》

Nous n'aurions plus la possibilité de développer notre personnalité; nous ne pourrions plus donner toute notre mesure, et notre propre existence tomberait dons l'ornière de lo routine. Comme Nietzsche l'a souligné: \& La connaissance du passé est toujours nécessaire, mais seulement pour servir ou présent et à l'ovenir; non pos pour affaiblir le présent, ou pour déraciner les puissances vigoureuses de l'existence future. $\gg$ II s'attaquait alors à cet historicisme vide de sens et à ce faux dogmatisme qui soutenaient que I'homme est déterminé par son histoire et son environnement, doctrines qui lui refuseraient en fait I'enthousiosme général dont il a besoin pour créer l'histoire et pour transformer son milieu ambiant, en fait son existence même.

Dans l'introduction de son livre \& Lo dualité conodienne $\gg, M$. Jean-C. Falordeau écrit: - Molgré la subdivision de notre poys en dix états provincioux distincts, malgré la diversité de ses régions géographiques et économiques, malgré lo diversité ethnique de sa population, malgré enfin la solidité de ses structures politiques transcontinentales, le grand fait de la vie conadienne est la coexistence de deux univers, l'un fronçais, l'autre anglais.

J'ai mentionné ouparavont le fait qu'un chongement peut être subjectif, c'est-à-dire qu'il peut s'exercer dons la conscience de l'individu ou des personnes dont l'agrégat forme une société, ou bien objectif dans la mesure où lo tronsformotion modifie les structures socioles, politiques et économiques du groupe. Si ces deux types de changement se produisent concurremment, il s'effectue alors nécessairement un troisième changement, celui-lò dans les relations existant entre l'individu et son groupe, dans les rapports et les liens qui unissent les hommes entre eux.

Je me propose de décrire ces trois changements comme je les vois, quoique très briève. ment, pour l'excellente roison que d'autres que moi se sont déjà livrés à une sembloble description; ensuite, je les exomineroi de deux points de vue différents: d'une part, du point de vue de l'homme d'affaires d'expression française et, d'autre part, du point de 
vue des milieux d'affaires d'expression anglaise. Incidemment, je ne m'attends à foire plaisir ni à la Chambre de Commerce de Montréal ni au Montreal Board of Trade.

$1^{\circ}$ L'aspect le plus remarquable de cette transformation de la psychologie québecoise est son aptitude à créer de nouveaux systèmes et de nouvelles structures, en particulier dans les domaines politiques et économiques. C'est dans ce sens que l'on peut dire que la Province est le triéátre d'une renaissance, du fait de son désir d'élargir le chomp de son activité et d'exploiter toutes les possibilités, quelle qu'en soit leur nature. Les institutions, relativement nouvelies, où s'affine notre connaissance des sciences socioles et de la pratique des affaires, ont multiplié les options possibles et l'intérêt des jeunes Québecois ne se concentre plus uniquement sur les carrières offertes par l'Eglise ou par les professioris libérales.

II n'y a rien de nouveau dans cette autocritique. Par contre, nous trouvons désormais une attitude entièrement nouvelle, à savoir celle des jeunes Conodiens fronçais qui ne réagissent plus pāssivement, comme leurs pères le faisaient, lorsqu'on leur ferme les portes ou nez. Cette jeunesse canadienne-française est bien décidée ò s'exprimer ovec une force, avec une énergie qui porte bien le sceau de leur conviction en de nouvelles formules, et ceci cux dépens. des systèmes et des institutions octuels. A cette ottitude, je n'ai rien à redire. II est parfaitement légitime que les jeunes gens qui sortent de nos universités cherchent à détruire les obstacles qui les empêchent d'atteindre les meilleurs postes de commande, tant dans le domaine de la finance que dans ceux de l'industrie et du commerce. Voilà en effet les nouveaux champs d'activités vers lesquels se concentre fondamentalement l'intérêt de notre jeunesse; or, les tensions et les difficultés commencent lorsque cette même jeunesse se retrouve face ò face ovec un milieu qui ne reconnaît pas, ou ne se rend pas compte, de sa compétence nouvelle.

Comme je l'ai dit, je n'ai pas d'objection en ce qui concerne l'énergie et la détermination qui caractérisent des efforts visant ò assurer une prépondérance et un pouvoir accrus sur. les affaires économiques. II est bon d'accorder à ces objectifs un caractère prédominant et, du fait de ce processus, de critiquer et de transformer les institutions actuelles. Cependant, nous ne pouvons pas, en notre âme et conscience, accepter le risque de la destruction de toute une société en flétrissont tout l'appareil de nos lois, notre forme de gouvernement et nos traditions politiques.

A bien des égards, la jeunesse du Québec doit trouver son inspiration dans ce véritable mentor que fut le brillant mathématicien, économiste, philosophe et homme politique que fut Marie Jean Antoine Nicolos Caritat, marquis de Condorcet. Elle pourra également trouver chez Condorcet une excellente leçon. Son optimisme, so foi dans la capocité de l'esprit humain à réaliser des progrès sans limite sous-entendaient l'existence d'une certaine stabilité au sein de la nation, si l'on désirait vraiment y promouvoir croissance et exponsion. En fait, on a dit que «la tragédie de Condorcet provenait du fait qu'il avait contribué à détruire le système d'outorité qui ourait justement permis l'opplication de ses idées \$. II participa en effet ou mouvement qui sopa en France l'outorité publique et so propre existence prit fin au zours des troubles et de la terreur qui s'ensuivirent.

Le progrès que nous recherchons ne peut être atteint par un renversement du système social qui existe au Québec. Dans le domaine politique il sera réalisé grâce ò la direction que nous donnerons des hommes dont la voleur est reconnue en droit constitutionnel, en histoire et en science politique; dans le monde des affaires nos objectifs seront atteints par 
un travoil laborieux et persévérant. Le recours aux fusils et à la révolution n'est pas la solution.

Lo Province n'a jomais dépensé pour l'instruction des sommes aussi considérables qu'ò I'heure actuelle. Celo signifie que nous sommes en train de procéder à d'énormes investissements pour la mise en valeur du copital humain, en vue de l'obtention de connaissances et de compétences techniques toujours plus nombreuses. La prochaine génération devra done avoir a so disposition des possibilités et des occosions véritab!es de montrer ce dont elle est capable. Si tel n'est pas le cas, nous pouvons nous attendre ò la frustration, ò l'anarchie et ò la fuite de nos meilleurs cerveaux vers d'outres pays. Les véritobles investissements auxquels se livre le gouvernement en vue de renouveler notre capital humain sont nécessaires ò la fois pour le développement de chaque individu et en tant que goronties d'un ovenir plus riche, spirituellement, culturellement et matériellement. Ces investissements représentsnt, de notre part, un acte de foi par les termes desquels nous exprimons notre conviction que les générations à venir seront en mesure d'utiliser leurs connaissances de façon à établir une paix et un ordre véritoble dans le monde. Nous ne pouvons plus revenir au monde des horizons d'onton.

Lo transformotion du Québec signifie que nous sommes capobles de prendre et que nous prenons, effectivement, consciemment, une part toujours croissante dans la rénovotion économique et industrielle de notre société. Techniquement et odministrotivement, nos possibilités de procéder à des changements s'accroissent constamment. Politiquement et économiquement, nous cherchons à obtenir la liberté de l'administration et de la direction de notre économie. L'essence du dilemne québecois relève de l'impatience de Condorcet. Nous savons ce que nous voulons et nous avons des moyens nouveaux qui nous permettront de réaliser nos objectifs mais il nous reste encore à tomber d'accord sur la rapidité de cette évolution de monière ò sotisfaire les éléments les plus cloirvoyonts de notre société, même si cela déplaît à nos réoctionnaires ou à nos révolutionnaires.

$2^{\circ}$ Etant donné que l'effet immédiot de nos projets concernant !'instruction publique est d'augmenter les investissements destinés à oméliorer et à accroitre notre capital humain, il s'ensuit que les possibilités de devenir plus créateur, plus productif et plus responsable que par le passé doivent également être amplifiées. Sinon, nous nous épuiserons dans une frustration et une déception dont nous n'ovons aucune idée.

Nous sommes ici ou coeur même du problème que posent les transformations survenant dans le monde réel. $Y$ auro-t-il place pour une direction intelligente et énergique, ou sein des grondes entreprises, pour une direction qui soit en mesure d'absorber le flot toujours croissant des diplômés qui sortent de nos universités et de nos écoles techniques?

Le science économique, telie que la connaissaient Adam Smith et John Stuort Mil!, concernoit surtout une épnque coroctérisée por des entreprises dirigées par des propriétaires uniques ou par des associés. Ni l'un ni l'autre n'était certain des avontages qu'offre la grande société ononyme. En fait, ce n'est qu'en surmontant de grondes difficultés que I'on pouvait constituer légaiement une compagnie en Grande-Bretagne après le Bubble Act de 1,20 et ce n'est que depuls environ une centaine d'années que l'hostilité à l'égard des sociétés anonymes a conmencé à diminuer. Cependant dès mii neuf cent, avec lo création des trusts United States Steel et Standard Oil, l'ère des sociétés-mères géontes, ayant des filiales ò trovers le monde entier, s'était amorcée d'une façon définitive. Nous ne pouvons pas revenir à l'époque où prédominaient les systèmes d'entreprises individuelles ou d'asso- 
ciations. Les géants sont là; ils sont efficients, puissonts, légalement éternels et suprêmement indépendants, souf en ce qui concerne les pressions politiques.

Face à ces structures industrielles apparemment complètes et adultes, dominée qu'elle est par les intérêts étrangers ou anglo-canadiens, il ne semble pas qu'il y ait beaucoup de place pour une nouvelle élite conodienne, réaliste et porfaitement préparée, d'expression française, désireuse de mettre en valeur et de développer une puissance industrielle de son cru. De plus, les entreprises familiales actuelles ne sont plus en mesure d'élargir leurs marchés et leurs sphères d'influence, et, de ce foit, ces entreprises se trouvent placées dans une situation où leur existence même est menacée par les grandes sociétés internationales et por leurs filiales, plus efficientes et plus solides du fait de leur puissance financière.

En un mot, donc, l'apparition de firmes nouvelles sur la scène nationale est devenue hasardeuse justement au moment où une nation nouvelle désire désespérément prendre son essor. Il est extrêmement coûteux d'entrer en concurrence avec la compagnie établie depuis longtemps et dont les reins sont solides; il est virtuellement impossible, pour des hommes nouveoux, de rossembler une épargne privée qui permette de procéder au lancement de grondes entreprises. Les impôts rendent possible le raffinement du capital humain, c'est-àdire la compétence et les connaissances de nos jeunes gens; par contre, le poids de la fiscalité diminue en même temps leurs possibilités d'épargner et d'accumuler les capitaux qu'exigeraient leurs aptitudes.

Cette situation n'est pas particulière au Québec. Le monde de l'entreprise moderne, I'univers de l'\&Organization Man», offre certes une sécurité mais certainement pas, du moins dans les débuts, la possibilité de se livrer à une activité particulièrement créatrice. Le sentiment très spécial de frustrotion du jeune diplômé canadien d'expression française est intensifiée par l'individualisme de ses traditions et de sa culture, et par son entrée forcée dans un monde et dans des formes d'organisation dont il n'a jamais fait partie jusqu'd présent.

$3^{\circ}$ Etont donné d'une part l'ougmentation fantastique de la compétence et du savoirfaire de nos jeunes gens, et, d'autre part, la réduction des possibilités de se lancer dans des initiatives et des affaires nouvelles, dans une société commerciale déjà développée, déjà orgonisée, il s'ensuit que les relations entre l'homme et son milieu ne peuvent pos, non plus, rester inchangées. On a déjà dit, et c'est exact, que le Québec n'a abandonné la philosophie victorienne du libéralisme genre «laissez-faire \ que depuis quelques années. Les très lourdes augmentations que connoissent nos dépenses dans les domaines scolaires et de l'enseignement technique, en vue de la construction de voies de communication, du fait de l'assurance-hospitalisotion, sous le poids des mesures sociales, sont surtout dues au désir de mettre à la disposition de notre peuple les mêmes services et les mêmes ovantoges dont d'outres provinces et d'outres poys profitent déjò depuis plusieurs dizaines d'onnées.

Les gouvernements ont un rôle valable et nécessaire ò jouer dans la mise en valeur des ressources humaines, dans la créotion d'un capital social sous la forme de voies de communicotion, et coetera, et dans la mise au point de programmes de recherche qui permettent de promouvoir ou mieux l'utilisation la plus efficiente possible de nos richesses et de nos industries. On ne peut pas s'attendre que les chefs d'entreprises petites et moyennes puissent vraiment concurrencer les sociétés colossoles qui caractérisent le monde d'aujourd'hui. Cette philosophie de l'individualisme économique était valable pendant les pre- 
mières décennies de la Révolution industrielle. De nos jours, l'impôt sur le revenu a diminué lo capacité d'épargner, donc d'investir de l'individu tandis que de nombreux dispositifs monopolistiques l'empêchent de faire son entrée dans la plupart des industries. Un peuple désireux d'ougmenter son standard de vie et son toux de croissance, un peuple décidé c̀ réduire le nombre de ses chômeurs se doit de faire usage de l'assistance puissante et de la coopérotion que les gouvernements peuvent lui accorder. Avant que ne soient mises en valeur, par exemple, la Gaspésie ou la Côte Nord, devons-nous ici, dans le Québec, attendre qu'oillent s'établir là-bas, volontairement, de petites affaires privées? Devrons-nous attendre pendant plusieurs générations la lente progression de ces petites entreprises jusqu'au stade de la maturité économique? Ou bien, ne devons-nous pos plutôt encourager activement l'essor de ces régions par une assistance finoncière constructive, par un système d'entreprises mixtes, et même au moyen de sociétés de la Couronne?

Le but de l'activité gouvernementale est de réduire le chômage et de faire disparatre les difficultés et la misère. Cet objectif n'est pos nécessairement celui que poursuivent les fllicles des sociétés étrongères ou même nos grandes sociétés, mais, la réalité le justifiant, cet objectif doit être otteint. Si celo signifie qu'une intervention supplémentaire du gouvernement est rendue nécessaire, directement ou indirectement, eh bien! nous interviendrons. Ce n'est pos là d'ailleurs une raison pour conclure immédiatement que lo liberté individuelle sera supprimée ou que nos dirigeants politiques ne sont qu'une bande de dictateurs communistes ou foscistes. Le libéralisme économique du dix-neuvième siècle n'aidera pas le Québec à prendre la place qui lui revient dans le concert actuel des sociétés industrialisées. L'opposition de nombreux hommes d'affaires est presque de noture réoctionnaire; or une telle attitude, fotalement, ne peut qu'être contrebalancée par des prises de position tou: oussi extrêmes de la part de nos révolutionnoires. Le Québec se trouve dans une situation de fermentation intellectuelle. C'est une province vivante, dont le cas constitue un défi passionnont. Tout en discutant les transformations que subissent les relations entre les gouvernements fédéral et provinciaux, oinsi que le rôle nouveou de ces derniers, nous sommes au moiris en mesure d'aller au devont des thèses les plus progressives de nos jeunes intellectuels, Ju fait d'un certain nombre de suggestions libérales modernes de notre cru.

En fait, la responsobilité de la croissonce économique n'o jamais été entièrement confiée à l'entreprise privée. Personnellement, je désire voir le secteur privé prendre toute l'ampleur dont il est copoble; par contre, on n'est pas socialiste parce que l'on est convaincu que le gouvernement a la responsabilité de promouvoir un supplément d'activité économique de nature ò faire diminuer le chômoge et à augmenter la production. Cela revient simplement à dire que, dans notre échelle des voleurs, nous plaçons le besoin de trovailler de l'homme et de la femme au-dessus de cette sacro-sainte inviolabilité des systemes établis d'organisation des affaires. Et ni la Chambre de Commerce du district de Montréol, ni le Montreal Boord of Trade, ne peuvent se permettre d'ignorer les tronsformotions en cours, ou de réogir passivement devant ces transformations.

Dans quelle mesure donnons-nous oujourd'hui aux jeunes gens frais émoulus de nos universités la possibilité de se mesurer ovec l'existence? Ets-ce que le seul débouché qui s'offre ò leurs tolents et ò leur énergie est d'entrer au service du gouvernement, ou bien le monde des affaires est-il prêt à leur donner la place ò laquelle leur compétence leur donne droit? Voilà véritablement le grand problème auquel les milieux d'affaires et le gouvernement doivent faire face; et l'avenir de la Province dépend de la solution que l'on souro trouver. En gordant d̀ l'esprit l'objectif fondamental que constitue lo recherche du 
plein emploi, je déclore qu'une saine gestion de l'économie de la Province de Québec exige, de la port du gouve nement et des milieux d'affaires, les attitudes suivantes:

1) Tant que subsiste le chômage, tant que notre croissance continuera d'être lente, le gouvernement provincial devra poursuivre sans relôche une politique agressive visant c̀ occroître les investissements et à promouvoir l'industrialisation. La Société Générale de Financement a tous les pouvoirs dont elle o besoin pour devenir une forme énorme dans lo province, tont en ce qui concerne les investissements en particulier que l'activité économique en général. La S.G.F. peut financer la création de nouvelles entreprises, promouvoir l'essor des compagnies en existence et assurer, si c'est nécessaire, la fusion de deux ou plusieurs sociźtés. Lo S.G.F. peut égolement contribuer à la mise en valeur des richesses naturelles du Québec en associant ses copitoux à ceux d'entreprises étrangères

L'une des préoccupotions majeures du gouvernement provincial est d'assurer un niveau suffisant d'investissements dans le Québec, car de ces investissements dépendent en défi. nitive nos toux de croissance, de chômage et de production. Nous ne pouvons pas nous permettre un recul de notre activité économiaue et, en cela, nous ne faisons que nous inspirer des réussites d'autres pays où une proportion croissante de l'ensemble de l'activité économique est le résultat d'une intervention gouvernementale à la recherche de la prospérité. En plus de la Société Générale de Financement, le gouvernement considère en ce moment un système de fonds de retraite qui viendro s'ajouter au flot des investissements. Aucune de ces mesures ne devrait soulever de critiques irréfléchies de la part du monde des offaires, car nos objectifs sont les mêmes que les siens: nous voulons maintenir notre activité économique à un niveau élevé et stable. Etant donné que nous sommes en train de fournir un «pool » toujours plus important de spécialistes et d'experts grôce à nos efforts dans le domaine de l'enseignement et de la préparation technique, nous avons par conséquent également la responsobilité de nous assurer que la masse de nos investissements est d'une ampleur telle que nous puissions donner du travail à ce nouveau copital humain.

Le désir du gouvernement est que les copitaux s'investissent dons l'ovenir de notre prosince. Nous nous rendons compte que lorsque le capital étranger vient s'établir au Québec, il nous fait, en queique sorte, le compliment de sovoir que nous avons l'aptitude et lo capacité d'utiliser ces fonds sagement et efficacement. Cet orgent vient ici dans le but de rapporter; mais, par lì même, il fait reculer le chômage, il met en valeur nos richesses et il nous verse des impôts. Le modeste profit qui part à l'étranger sous la forme d'intérêts ou de dividendes n'est qu'un prix bien minime en comparaison de l'accélération de notre croissance et de notre activité économique.

On semble vouloir faire grond cas de la perte de souveraineté que de telles opérations entrcineraient. Je ne suis pas d'accord avec cette objection. Les capitaux étrangers acceptent les conditions sociales et l'atmosphère politique dans lesquelles ils doivent fonctionner et les décisions des dirigeants d'entreprises ne peuvent pas aller et ne vont pas à l'encontre des désirs exprimés par les gouvernements locaux. Dans le cas de l'acquisition récente d'une brosserie, je ne peux voir que fort peu de différences entre les décisions que prenait l'ancienne direction et celles que pourront prendre les nouveaux propriétaires. En fait, la plupart des décisions sont prises dons cette industrie par les outorités provinciales qui règlementent l'emballage, la distribution, la publicité, les méthodes de vente, etc. L'ospect fondamental de cette acquisition, comme de n'importe quel achat de compognie, c'est le réinvestissement des capitoux supplémentaires qui en résultent, de ces capitoux 
Relations Industrielles, vol. 19 , No 3

qui tombent soudainement entre les mains de Canadiens. Ces sommes seront-elles dépensées sous la forme de résidences sur la Côte d'Azur, seront-elles investies dans des pays soucieux d'attirer des capitaux, ou seront-el!es placées ici, au Canada, de façon ò donner du travail à des Conadiens, à utiliser des matériaux canadiens et à augmenter la production conadienne de biens et services.

2) M. Gérard Filion a parcouru la Province: partout il a déclaré que l'industrie québecoise doit prendre de l'ampleur.

* Des secteurs complets de l'industrie québécoise sont à réorganiser. LI faudra, d'ici une génération, procéder à de nombreuses fusions d'entreprises. II va falloir que les propriétaires actuels se mettent dans la tête qu'il vaut mieux être actionnaire minoritaire d'une grande entreprise prospère que d'être l'unique propriétaire d'une petite entreprise appelée à disparaître. Et pour cela, ils devront s'habituer à travailler en équipe. Si cette reconversion n'est pas faite d'ici une génération, une bonne partie de l'industrie de la chaussure, de l'alimentation, du meuble, de ia confection, du petit outillage sera absorbée par de grandes sociétés américaines ou disparaîtra. Un secteur important de l'industrie québécoise vit encore ou 19ième siècle. II faudra s'habituer à vivre au 20 ième siècle et se préparer à vivre au 2 lième siècle »

Je suis en plein accord avec cette déclaration et je demande à tous les hommes d'affaires d'expression française de bien noter ces paroles.

Nombreux sont les facteurs qui contribuent d̀ donner aux grondes entreprises leur efficience supérieure, y compris la productivité des machines par rapport à la maind'oeuvre, les ovantoges que confèrent des services de recherche, les techniques nouvelles et les méthodes ultra-modernes d'administration. Si nous sommes décidés à obtenir notre part des marchés canadiens et de l'étranger, nous devons adopter la philosophie qui est la base même du succès. Nous ne pouvons pas nous renfermer sur nous-mêmes et espérer que nous allons survivre en même temps. Nous ne pouvons pas desservir seulement nos proches marchés locaux et attendre ensuite cette expansion dont la Province a besoin. Nous devons nous rappeler également que nous ne pouvons demander infiniment à nos clients de payer un prix plus élevé que ne l'exige la concurrence car alors c'est le consommateur qui défraie le coût de notre incopacité à concurrencer sur le marché.

Plus vous vous acharnez à protéger vos débouchés, plus grande sera la part d'activité que vous abandonnerez à vos gouvernements et plus prononcé sera le penchant socialiste que vous craignez tous chez vos dirigeants politiques.

La responsabilité de cette Chambre consistera à instiller chez tous ses membres une prise de conscience des besoins que nous éprouvons, à savoir la nécessité de progresser et d'accroître toutes nos industries, si nous voulons vraiment employer nos gens d'une façon utile et créatrice La croissance est la note dominante de toute l'activité économique, et non la perpétuation d'une petite sphère tranquille d'influence.

Tous les efforts déployés par votre gouvernement dans le but de promouvoir la relance économique du Québec seront inutiles si les hommes d'affaires eux-mêmes n'acceptent pas ce principe de la croissance et ne s'en vont pas à la recherche du profit en progressant et en s'emparant de nouveaux marchés. Combien $y$-a-t-il par exemple de courtiers 
conadiens-français en valeurs mobilières qui possèdent des bureaux ò Winnipeg et à Vancouver? Aucun. Quand nos firmes envoient-elles des vendeurs à travers le pays afin d'écouler les obligations du gouvernement québecois et de l'Hydro-Québec? Très rarement. A vrai dire, ce n'est peut-être pos juste de nommer une industrie en particulier alors que tant d'autres se trouvent dons le même cas; par contre, c'est une industrie que je connais fort bien et de laquelle nous attendons beaucoup plus que par le possé

3) Si lo Chambre de Commerce doit instiller chez ses membres une confiance accrue dans le caractère nécessaire de notre progrès et de notre expansion, ceci dans le but d'assurer une utilisotion valable de toutes nos richesses humaines et matérielles, le Board of Trade, par contre, se doit également de reconnaître les changements survenus dans le Québec en reconnaissont que le rôle accru du gouvernement ne signifie pas l'avènement d'un état totalitaire. Nous avons tous foi et nous chérissons tous cet idéal que sont la liberté individuelle et l'entreprise privée, mais par contre, cela fait longtemps que nous avons appris que la recherche étroite du profit ne peut répondre à tous les besoins de notre société, que ces besoins soient d'ordre social sous la forme de garanties contre la misère et la détresse, ou qu'ils soient d'ordre économique, sous la forme d'une assurance, de notro croissance et de notre prospérité! En fait, une telle philosophie n'a donné lieu qu'ò une succession de périodes de prospérité et de dépression, et le rôle du gouvernement n'a pris toute son ampleur que dans la mesure où il était devenu nécessaire de contrôler et d'amortir la rigueur de ces cycles. L'espoir des communistes en la décadence de l'Occident repose dans leur conviction que le système de l'entreprise privée, sans direction aucune, ne peut qu'entraîner inévitablement de sévères dépressions qui provoqueront en définitive son effondrement.

L'activité du gouvernement peut être constructive et d'une grande assistance. Cette activité est absolument nécessaire si l'on tient compte des problèmes auxquels le Québec doit faire face en ce moment. Cette activité, comme je l'ai dit au commencement, implique une tronsformation des relations qui unissent l'homme et la Société, parce qu'il y a eu changement aussi bien chez l'homme lui-même que dans le monde où il évolue. De même que les hommes d'affaires d'expression française doivent reconnaître avec plus de conviction que jamais le déii que posent le problème de notre expansion et la dynamique de notre progrès, de même les hommes d'affaires d'expression anglaise doivent-ils reconnaître la justification du rôle plus grand que doit jouer le gouvernement dans notre économie. Une acceptation immédiate de cette évolution contribuero énormément ò réduire le besoin d'une intervention gouvernementale à mesure que nous progressons vers une coopération, un optimisme et une confiance plus grande que jamais. Par contre, des critiques irréfléchies et irraisonnées de tout ce qui constitue la politique gouvernementale ne contribueront qu'à aggrover les possibilités de conflits et à durcir les positions des différents groupes qui forment notre société.

Telle n'est pos la situation actuelle et je suis heureux de le déclarer. En dépit des monifestations extrémistes, c'est avec rapidité que la Province est en train de progresser vers une ère nouvelle caractérisée par une prospérité et une expansion toujours plus grandes. $\mathrm{Si}$ nous cherchons tous à découvrir la signification des changements dont nous sommes à la fois les témoins et les acteurs, si nous cherchons à les comprendre et à les accepter, nous atteindrons inévitablement ce grand but que sont la paix et l'harmonie entre nos deux. peuples. 
Comme a écrit le T.R.P. Louis-M. Régis, O.P.: \& $C^{\prime}$ est dans le respect de cette hiérarchie des voleurs que notre pays se donnera simultanément la paix et la prospérité, au sein d'une unité politique jalousement fidèle ò préserver et à favoriser les deux tradi. tions culturelles qui constituent notre originalité ou milieu d'un monde voué à la monotonie de la standardisation.

\section{RECENSIONS - BOOK REVIEWS}

Lois techniques et économiques de la production par R. Frisch, Dunad, Paris, 1963, 378 poges.

Selon l'auteur, les lois de production représentent la façon dont le ou les produits répondent aux modifications quantitatives de leurs facteurs. De plus, on parle d'anolyse économique quand les prix du marché entrent en ligne de compte. Dans le présent ouvrage, l'idée dominante est celle d'optimum et le raisonnement procède de la logique fondamentale des lois de production.

Le développement des institutions sociales et politiques a fait des deux hypothèses que sont la libre concurrence ou le monopole, un dilemme formel dont les termes répondent de moins en moins à la réalité des faits. Afin de rendre davantage compte de la réalité, l'auteur procède à une classification des principaux types de stratégie que révèlent l'observation. II distingue ainsi deux grandes catégories qu'il intitule stratégies de fixation et stratégies de négociation. Pormi les principaux types de stratégies de fixation, l'auteur considère l'adaptation (autonome) par la quantité, l'adaptation stochastique par le prix, l'adaptation par le prix ou par la quantité soumise à l'élasticité, le formulateur d'option et enfin, I'acquéreur d'option. De plus, il onalyse distinctement les principaux objectifs suivants: minimisation des coûts, maximisation de la production, maximisation du profit et enfin, optimalisation du profit.

Dans une première partie, le professeur Frisch traite de divers concepts: qu'est-ce que la production? facteurs de production; technique de production et différents types de théorie de la production. Dans les trois autres parties, il anolyse trois situations différentes: production instantanée simple ne comportant que des facteurs continus, production instontanée simple avec facteurs limitatifs et production multiple. Dans chacune de ces parties, l'auteur considère ò tour de rôle l'aspect technique et l'aspect économique. Dans la section technique, il utilise des concepts bien connus tels que productivités marginales, accélérations de production, productivités moyennes; élasticités moyennes et, entre autres, un concept relativement nouveau, soit le coefficient de conversion. La dernière partie expose les éléments d'une théorie dynamique de la production.

Le présent ouvrage représente probablement une contribution originale à la théorie économique de l'entreprise. II faut, de plus, souligner le caractère très scientifique de ce livre de même que la clarté de l'exposé en général. L'expression en termes mathématiques pour une large part en rend cependant la lecture quelque peu difficile pour les non-initiés aux mathématiques.

Bertrand Belzile

Comment administrer une entreprise. Publication du Ministère du Commerce, Ottawa (Canada), 1963. 120 pp.

Ce petit fascicule n'est pas un traité d'administration des grandes entreprises, mais un recueil de conseils protiques adressés à ceux qui exploitent une petite entreprise ou songent à en fonder une. Ce recueil traite d'abord de l'importance de la petite entreprise dans l'économie et les responsabilités qui incombent au propriétaire. Au deuxième chapitre, on distingue et définit les formes légales que peuvent prendre les entreprises.

Le troisième chopitre est un relevé des facteurs dont il faut tenir compte au cours du lancement d'un atelier ou d'une usine et d'un commerce de détail, en particulier. L'accès aux matières premières, la maind'oeurre, les conditions de vie dans la localité, les moyens de transport, les zones de marchés, les impôts locaux, le choix d'une 